\title{
Implementación de la Industria 4.0 en los procesos de distribución de las principales empresas lácteas: Tulancingo, Hidalgo
}

\author{
Implementation of Industry 4.0 in distribution processes within the main dairy companies : \\ Tulancingo, Hidalgo
}

Aline Paola Arriaga-Atanacio ${ }^{a}$, Ruth Ortiz-Zarco ${ }^{b}$

\begin{abstract}
:
The present research explores the degree of implementation of industry 4.0 in the distribution processes of a group of dairy companies, located in the municipality of Tulancingo in the State of Hidalgo, In the same way, the factors that encourage the technological lag of the selected companies are identified, and the opportunities that an enterprise with high technological capacity has within the region of study are identified.
\end{abstract}

\section{Keywords:}

Distribution, Fourth Industrial Revolution, Industry 4.0, Technological Implementation

\section{Resumen:}

La presente investigación explora el grado de implementación de la industria 4.0 en los procesos de distribución de un grupo de empresas de la industria láctea, ubicadas en el municipio de Tulancingo en el Estado de Hidalgo, de igual forma se determinan los factores que propician el rezago tecnológico de las empresas seleccionadas, y se identifican las oportunidades que posee una empresa con elevada capacidad tecnológica dentro de la región de estudio. El resultado denota la presencia de factores externos que retrasan e imposibilitan el desarrollo tecnológico del sector, aminorando su competitividad.

\section{Palabras Clave:}

Distribución, Cuarta Revolución Industrial, Industria 4.0, Implementación Tecnológica

\section{Introducción}

El sector empresarial a lo largo del tiempo ha enfrentado constantes desafíos, entre ellos los periodos de fuertes cambios tecnológicos denominados "revoluciones tecnológicas" las cuales, han desempeñado un papel determinante en el avance industrial y tecnológico dentro de los procesos de fabricación y distribución. En 1760 se vivió la primera revolución industrial, la segunda revolución industrial inició en el año 1850, posteriormente con el surgimiento de la computadora y el internet surgió la tercera revolución industrial en el año 1960; actualmente se vive la cuarta revolución industrial, que consiste en una transformación digital de la industria, la cual ha marcado un cambio en la forma en que las empresas deben rediseñarse debido al constante desarrollo de capacidades digitales las cuales se enlazan a las necesidades de la población y, ¿Por qué no del mundo?, ante ello surge el siguiente cuestionamiento: ¿Qué tan necesaria es la implementación de los avances tecnológicos propios de la industria 4.0 en las empresas, como medida para impulsar la competitividad y la supervivencia?.

Este trabajo busca responder la pregunta anterior, mediante el análisis de un grupo de empresas lácteas ubicadas en Tulancingo Hidalgo, ante la implementación de la industria 4.0 en sus procesos productivos y de distribución; las empresas seleccionadas para el estudio son: Márquez Agroindustria de Hidalgo, Lácteos Elena,

\footnotetext{
a Autor, Licenciatura en Comercio Exterior, Universidad Autónoma del Estado de Hidalgo-Instituto de Ciencias Económico AdministrativasUAEH, https://orcid.org/0000-0002-3676-1069 E-mail: ar277790@uaeh.edu.mx

${ }^{b}$ Coautor de Correspondencia, Licenciatura en Comercio Exterior, Universidad Autónoma del Estado de Hidalgo-Instituto de Ciencias Económico Administrativas-UAEH, https:/lorcid.org/_0000-0002-7050-1069 E-mail: ruth_ortiz@uaeh.edu.mx
} 
Cremería los Andes, KRENDYC y Los Luna; los factores que estipularon la selección de empresas son: el espacio físico de la fábrica, las características del proceso de producción, la distribución desde almacén a punto de venta (propio o local externo a la empresa), y el uso de tecnología en sus procesos de distribución. Las empresas seleccionadas enfrentan la necesidad de adaptarse a la nueva revolución tecnológica, conocida como Industria 4.0 o El internet de las cosas. "La Industria 4.0 implica la combinación de técnicas avanzadas de producción y operaciones con tecnologías inteligentes que se integrarán en las organizaciones, las personas y los activos (Deloitte Touche Tohmatsu Limited, 2017)". 1

Ante estas nuevas necesidades tecnológicas y el surgimiento de la cuarta revolución, la industria láctea requiere modificaciones inteligentes, desafortunadamente en algunos casos las empresas se ven imposibilitadas para la consumación de dichos cambios, es aquí donde surge la pregunta ¿Las empresas que no han logrado implementar la industria 4.0, realmente son incapaces de adoptar el desarrollo derivado de esta nueva revolución? o ¿Existe un factor externo que les impide realizar dichos cambios?, para poder responder a dichos cuestionamientos se identificaron los factores de riesgo, las ventajas y desventajas que presentan las principales empresas lácteas de Tulancingo, así como los factores que imposibilitan o retrasan la ejecución de la Industria 4.0 en base a ello, se fundamentará la necesidad de la implementación de los avances tecnológicos de la actual revolución industrial.

Para representar las necesidades de implementación ante los rezagos tecnológicos de las empresas lácteas de Tulancingo, se realiza un análisis comparativo entre las siguientes entidades federativas: Hidalgo, Estado de México, Chiapas, Veracruz, Jalisco, Guerrero, Tabasco, Nayarit, San Luis Potosí y Tamaulipas, así como la importancia nacional que representa la implementación de la industria 4.0 en los procesos de distribución de las empresas lácteas como medida de competitividad e innovación.

Se realizará un análisis por entidad federativa que abarca todo el país, basado en la posición que ocupa en las siguientes áreas evaluadas:

- Infraestructura académica y de investigación

- Formación de recursos humanos.

- Personal docente y de investigación.

- Inversión en Ciencia, Tecnología e Innovación (CTI).

- Tecnologías de la información y comunicaciones (TIC's).

- Productividad científica e innovadora.
Se destacan las posiciones ocupadas por el Estado de México y el Estado de Hidalgo, a fin de resaltar el estatus de las entidades federativas que han integrado a sus procesos los cambios pertinentes en sus procesos productivos, como estrategia de competencia en el marco de la cuarta revolución industrial. Surge una pregunta más, ¿Implementar la industria 4.0 a los procesos de distribución es el único factor necesario para ser una empresa competitiva, o es solo un paso para sobrevivir en un ambiente de competencia? para dar respuesta al planteamiento anterior se indaga el contexto tecnológico al que se están enfrentando y se enfrentarán en los próximos años las empresas del sector lácteo de Tulancingo, poniendo especial atención a los factores que entorpecen la implementación de la industria 4.0, así como los retos y oportunidades que conlleva el desarrollo tecnológico para las empresas analizadas, a quienes se aplicó una encuesta como herramienta de evaluación interna de los estándares tecnológicos con los que operan sus servicios de distribución dentro del estado y de los elementos que determinan la adopción de la industria 4.0 en estas empresas.

\section{REVISIÓN BIBLIOGRÁFICA}

Los estudios que analizan la implementación de la industria 4.0 en el sector lácteo son escasos, no obstante, en este apartado se presentan las aportaciones de documentos que anteceden a este; entre ellos los pertenecientes a: Del Valle (1986); Cuevas (2007); Santillán (2009); Cervantes (2013); Loera (2017); Cardona (2017); Huartos (2019).

María del Carmen en el año 1986,analizó las industrias lácteas fronterizas, así como su desarrollo y limitaciones; presentó una caracterización del proceso de producción y consumo de leche para resaltar los problemas sobresalientes, concluyó que en las ciudades fronterizas se presenta un volumen representativo de importaciones de este alimento debido a la desarticulación de la zona con los centros productores, por no haber restricciones para su importación y por las ventajas en cuanto a calidad, (Del Valle, 1986). 2

De igual manera, en el año 2007 Cuevas analizó el desempeño de la cadena productiva de la leche de vaca en el estado de Hidalgo, e identificó los factores críticos que presentan ante la intervención de la industria 4.0, mediante un muestreo de proporciones de varianza máxima y entrevistas concluyó que los factores críticos de cada eslabón y sus impactos transversales de la industria en Hidalgo, (Cuevas Reyes, 2007). 3

Así mismo, Santillán en el año 2007 identificó los principales factores críticos y demandas tecnológicas y políticas públicas para mejorar la competitividad de la cadena agroalimentaria de la leche de vaca en el Estado 
de Hidalgo, se determinó que gran parte de las demandas de tecnología y conocimientos pueden ser cubiertas por la oferta disponible, por lo que concluye que la mayor parte se dedicará a desarrollar políticas públicas, formar a los productores y desarrollar un proceso de transferencia de tecnología, para garantizar que la tecnología generada en los centros e instituciones de investigación y educación llegue a los eslabones de la cadena agroalimentaria desde la leche de vaca en el Estado de Hidalgo, (Romero Santillán, et al., 2009). 4

Cervantes (2013), realizó un estudio para comparar la calidad estándar de la leche entre cuencas para determinar cuál de ellas presenta una mejor calidad o, en dado caso, una menor deficiencia, el estudio se extiende para el Estado de Hidalgo, Tizayuca y el Valle del Mezquital y concluye mediante un cotejo de las entidades que la leche de Hidalgo con las normas internacionales (comparación al exterior), es excelente en rendimiento industrial, con deficiente sanidad, y probablemente adulterada con agua sin embargo, (Cervantes Escotoa, et al., 2013). 5

Después del estudio de Cervantes ante la calidad de leche en Hidalgo, podemos interpretar que es un estado que puede competir ante normas internacionales, por lo que Loera (2017), realizó un estudio de la industria lechera en México, los parámetros de la producción de leche y abasto de un mercado interno analizando la situación de la industria lechera nacional, el estudio se extiende a las 32 entidades federativas y concluye que existe una brecha entre producción y demanda interna del lácteo la cual brinda la oportunidad para aumentar la producción y sustituir importaciones en México, (Loera \& Banda, 2017). 6

Cardona (2017), en su libro titulado Innovación en los procesos logísticos: Retos locales frente al desarrollo global, busca comprender la incidencia de los indicadores de eficiencia logística empresarial (KPI) y los indicadores de desempeño logístico (LPI) enfocados en el componente de las tecnologías de la información y comunicación para la distribución de empresas en el departamento del Atlántico. Basado en datos empíricos producto de un levantamiento de datos a través de encuestas dirigidas a empresarios y directivos en logística, concluyó que basado en los resultados del trabajo se alcanza a entender algunas dinámicas por las cuales se realiza la gestión de distribución, así como su medición, evidenciando además el bajo nivel de utilización de tic en la gestión logística, (Cardona Arbeláez, et al., 2017). 7

Huartos (2019), en su trabajo resalta y demuestra la importancia del aprovechamiento de la logística 4.0 y sus aportes al proceso de distribución de última milla, desarrollándolo con un enfoque investigativo y descriptivo que ilustra cronológicamente el panorama actual del proceso de entrega de mercancías y su evolución como proceso logístico que genera ventajas competitivas con la aplicabilidad de nuevas tecnologías, en un mundo más interconectado, y concluyó que la logística 4.0 cobra importancia en los procesos de distribución de última milla y brinda alternativas de aplicabilidad vanguardistas con la inclusión de inteligencia artificial y sistemas de información que incrementan la infraestructura y capacidad de respuesta de las empresas de transporte, haciendo una sinergia efectiva entre el recurso humano y los recursos de automatización, (Importancia del aprovechamiento de la logística 4.0 y sus aportes al proceso de distribución de última milla, 2019) .8

Con la finalidad de representar la necesidad de innovar e implementar tecnologías de la industria 4.0, dentro de las industrias lácteas del estado de Hidalgo, se presentan los resultados de algunos documentos; Oliván (2014); Anastacio (2015); Medina (2016); Schwab (2016); Yuval (2017); Ynzunza (2017); Anoor (2017); Taliaferro (2018); Bearzotti (2018); Silva (2018); Basco (2018); Arteaga (2018); Calatayud (2019).

Oliván (2014), realizó un estudio de la cuarta revolución industrial con el fin de afrontar la interrogante de qué papel ha de jugar la cultura en las ciudades del futuro desde un punto de vista materialista, construyendo un relato en torno a los tres ejes orgánicos donde los cambios van a ser más profundos: el trabajo, la gobernanza y las empresas, el estudio de extiende a las 32 entidades federativas y concluye que la cuarta revolución industrial va a multiplicar la productividad de las empresas, va a acortar los ciclos de innovación/ganancia, favoreciendo a los emprendedores frente a los rentistas, (Oliván Cortés, 2014). 9

Anastacio (2015), analizó la dinámica del uso de innovaciones pecuarias y su incidencia en las ganancias del sistema de producción de lechería familiar en el Valle del Mezquital para contribuir con elementos en la toma de decisiones e incentivar la productividad de este sistema concluyendo que la producción de leche en el Valle del Mezquital cuenta con factores que contribuyen a la competitividad y cuentan con una mayor productividad de leche por vaca, (Espejel García, et al., 2015). 10

Por el contrario, Medina (2016), analizó el financiamiento de la ciencia, la tecnología y la innovación en las regiones de México, analizando el impacto de los tres principales programas de financiamiento del sector de ciencia, tecnología e innovación en las 32 entidades federativas concluyendo que el financiamiento ha jugado un papel importante en la creación de condiciones para el desarrollo y competitividad de las regiones, pero no de manera equilibrada entre las entidades federativas, (Medina Rivera \& Villegas Valladares, 2016). 11

Schwab (2016), en su libro titulado "La cuarta revolución industrial" describe las características clave de la nueva 
revolución tecnológica y resalta las oportunidades y dilemas que ésta plantea, establece las nuevas formas de colaboración y gobernabilidad, acompañadas de una narrativa positiva y compartida, pueden moldear la cuarta revolución industrial para beneficio de todos y concluye con el pensamiento de que si aceptamos la responsabilidad colectiva de crear un futuro en el que la innovación y la tecnología sirvan a las personas, podremos llevar a la humanidad a nuevos niveles de conciencia moral, (SCHWAB, 2016). 12

Así mismo, Ynzunza (2017), exploró el entorno tecnológico, así como las implicaciones y perspectivas futuras de la industria 4.0 en los diversos sectores económicos de México, el análisis se extiende a las 32 entidades federativas y concluye que los componentes principales giran en torno a los sistemas ciber físicos, las máquina y productos inteligentes, el internet de las cosas, el internet de los servicios, así como las fábricas y ciudades inteligentes, (Ynzunza Cortés, et al., 2017). 13 Cohen Yuval (2017), plantea cómo se espera que ocurran las transformaciones correspondientes a la industria 4.0. Se presenta dentro del documento un futuro mapa de estado de los paradigmas de montaje como efecto de la integración con los principios de dicha industria y concluye proporcionando una arquitectura general para implementar estos principios en los sistemas de montaje existentes, (Cohen, et al., 2017). 14

Taliaferro (2018), examinó la evolución de la funcionalidad DC y exploró las aplicaciones de las tecnologías emergentes impulsadas por la Industria 4.0 para permitir un centro de distribución más flexible, adaptable y productivo en los diversos sectores económicos concluyendo que dentro de la cadena de suministro, las tecnologías de la industria 4.0 pueden permitir a las organizaciones planificar mejor la demanda y gestionar con mayor precisión el inventario dentro del centro de distribución. Esto se debe, en parte, a la mayor trazabilidad de los productos que permiten las tecnologías conectadas, (Taliaferro, et al., 2018). 15

Para realizar una comparativa de las capacidades dinámicas en las MIPYMES para la adaptabilidad de la industria 4.0 en Hidalgo, Silva (2019), abordó las necesidades digitales en las pymes para traducirlas en capacidades educativas requeridas de los futuros egresados de ingenierías que podrán ayudar a la formación de ingenieros 4.0, el análisis se extiende a las 32 entidades federativas concluyendo que se necesitan desarrollar capacidades digitales como computación de la nube, internet de las cosas y manufactura aditiva para satisfacer la futura demanda de la industria en las MIPYMES como medida de competitividad, (Silva Casas \& Estrada Dominguez, 2019). 16

A su vez, Bearzotti (2018), aborda los desafíos asociados con la adopción de la Industria 4.0 en la gestión de la cadena de suministro en los diversos sectores económicos, concluyendo que la cuarta revolución industrial ha comenzado a desarrollarse y las organizaciones de este tiempo les toca el desafío de ser las protagonistas del cambio, conociendo los riesgos a los cuales se exponen por ser actores de esta transición. Sin embargo, la no adaptación a estos cambios es una condena a la pérdida de competitividad, (Bearzotti, 2017). 17

Basco (2018), explora el impacto de las nuevas tecnologías sobre la industria manufacturera, el trabajo, las cadenas globales de valor y el comercio, a su vez se analiza las oportunidades y los desafíos asociados a esta Cuarta Revolución Industrial concluyendo que la fábrica inteligente sintetiza estas transformaciones logrando un alto nivel de automatización, de integración vertical y horizontal en su cadena de valor y de flexibilidad en la producción, (Baco, et al., 2018). 18

Arteaga (2018), en su documento de trabajo denominado La cuarta revolución industrial (4RI): un enfoque de seguridad nacional, revela un desfase en la adaptación española respecto a los principales competidores o la media europea en casi todos los requisitos necesarios para afrontar con garantías la 4RI, concluye postulando las carencias que en conjunto representan un reto sistémico para la industria española, concluyó que, de no remediarse a tiempo el reto sistémico, dañará gravemente la prosperidad nacional, (Arteaga, 2018). 19

Así mismo, Calatayud (2019), realizó un estudio para identificar la experiencia, lecciones aprendidas y mejores prácticas en la transformación digital de la cadena de suministro en economías avanzadas, evaluar el nivel de preparación de América Latina para encarar dicho proceso de transformación, y presentar una serie de recomendaciones para acelerar este proceso en la región concluyendo que por el potencial disruptivo del nuevo contexto productivo internacional, la transformación digital debería ser un aspecto de central preocupación para los gobiernos de la región, sus empresas líderes y sus pequeñas y medianas empresas, (Calatayud \& Katz, 2019). 20

\section{DESARROLLO}

A fin de cumplir con el objetivo de la presente investigación, se detalla la metodología a seguir en el análisis de las empresas lácteas seleccionadas. En primer instancia, se seleccionó a las cinco empresas de estudio, las cuales fueron seleccionadas de acuerdo a su capacidad de producción, el tamaño de sus instalaciones, la distribución de productos a un punto de venta así como la implementación tecnológica en sus procesos de distribución, posteriormente se llevó a cabo una visita a las instalaciones esto con el fin de visualizar la capacidad de distribución con que cuentan así como los dispositivos con que se desempeña, en este punto se reunieron las 
evidencias descritas de los avances tecnológicos de las empresas, posteriormente se recurrió a una llamada telefónica para evaluar los conocimientos con que se cuenta en cada empresa respecto a la industria 4.0 y su implementación, es en este punto donde se demostró que es necesario capacitar al personal de las industrias lecheras en el Estado de Hidalgo, con conocimientos en uso y manejo de tecnología así como dispositivos automatizados con el propósito de optimizar la producción y la competitividad de éstas.

Al recabar los datos anteriormente mencionados se procedió al análisis de los mismos y mediante éste la elaboración del cuestionario que será aplicado como medio comparativo y de evaluación para fines de esta investigación, por ello se presentan trece preguntas enfocadas en el conocimiento de tecnologías, el uso de dispositivos como rastreo y monitoreo de unidades, sensores, la comunicación que existe entre las áreas de labor y sus procesos de distribución, la interfaz con que se cuenta o si ésta es nula en la empresa, así mismo se evalúa su capacidad para implementar tecnologías a futuro como medio de competitividad y distinción de estas empresas.

El diseño del cuestionario (Anexo 1) se sustenta en los lineamientos de caja de herramientas propuesta por Hartmut Rauen, Gerente general interino del Verband Deutscher Machinen und Anlagenbau (VDMA), la elección de esta propuesta se basa en su concepción de los avances tecnológicos en el área de producción y distribución, así como la integración de los rubros descritos a continuación:

- Integración de sensores y actores

- Comunicación y conectividad:

- Almacenamiento de datos e intercambio de información

- Monitoreo del área de distribución

- Servicios de Tecnologías e Información (STI) asociados a los productos

- Modelos de negocio para los productos

Para conocer el nivel en que la empresa ha incursionado en la industria 4.0, se lleva a cabo un análisis del nivel de implementación tecnológica en los siguientes apartados:

- Procesamiento de datos en la producción

- Comunicación máquina a máquina

- Integración en red con la distribución a nivel de la empresa

- Infraestructura de tecnologías de información y telecomunicación

- Interfaces personas-máquinas

- Eficiencia en la producción de lotes pequeños

Al realizar el análisis así como el cuestionario pertinente, se pudo observar que las empresas seleccionadas para fines de este estudio, no cuentan con conocimiento actualizado sobre el uso-manejo de las diversas tecnologías aplicables a sus procesos de distribución, es decir, el nulo conocimiento de las alternativas tecnológicas ha imposibilitado el desarrollo e incursión de la industria 4.0 en este sector, a su vez, tan solo el $40 \%$ de las empresas tienen al menos 5 personas jóvenes laborando, los cuales cuentan con habilidades para el uso de dispositivos electrónicos con acceso a internet $y$, aunque no cuentan con conocimiento de herramientas de transporte 4.0, han laborado con dispositivos de rastreo y plataformas que han posibilitado a las empresas el acceso a nuevos mercados $y$, por lo tanto, a implementar tecnologías a sus procesos.

Begoña Cristeto, Secretaria General de Industria y Pyme, en el Foro "La transformación digital: la oportunidad para la industria española", dijo: "El futuro de la industria será digital o no será", (Cámara de Comercio de España, 2017). Esto quiere decir que ya no existe otra alternativa para las empresas que adoptar los avances tecnológicos, invirtiendo en innovaciones e investigaciones para transformarse en una empresa digital e incursionar en la Industria 4.0 para ser competitiva; disminuyendo costos, aumentando su productividad y eficiencia, optimización y flexibilidad en sus procesos, envío e intercomunicación de datos en tiempo real, digitalización y automatización de maquinaria, a su vez, sino se implementan estos avances la empresa corre el riesgo de no satisfacer las necesidades de su mercado y, en este caso, perder la posibilidad de seguir compitiendo y posiblemente desaparecer. 21

No obstante, Hidalgo se ubica en el lugar 20 por su aportación al PIB total nacional; lo anterior se explica por los obstáculos que enfrentan las empresas hidalguenses, como el bajo nivel de encadenamiento productivo, la dificultad para acceder al financiamiento, la falta de coordinación con el sector gubernamental y la carencia de innovación tecnológica en sus procesos de producción y distribución, lo que repercute en la productividad y en que el Estado se encuentre en la posición número 20 en materia de competitividad.

México se encuentra en el lugar 51, de acuerdo con el reporte Global de Competitividad (edición 2016-2017) del Foro Económico Mundial, (WeFORUM (Schwab, K.), 2017). El rezago que en materia de competitividad existe en el país se reproduce en Hidalgo que, según el Índice de Competitividad Estatal 2016 del Instituto Mexicano para la Competitividad (IMCO), se posicionó en el lugar 20 en 2014, después de ocupar las posiciones 24 en 2012 y 23 en 2010, (IMCO, 2016). 22,23

Esto quiere decir que las posibilidades de implementación en la industria 4.0 a nivel estado son bajas, aunque no imposibles, el grado de rezago en materia de competitividad y los bajos recursos económicos son tan solo una parte de la problemática, el estudio realizado a las empresas lácteas en la región de Tulancingo, refleja 
que su implementación de esta industria es en mayor parte, nula, se ha comprobado mediante la encuesta telefónica, que las empresas desconocen alternativas de implementación tecnológica que no conciban para ellos un gasto económico imposible, esto se debe principalmente al desconocimiento en materia tecnológica y el uso de dispositivos electrónicos para la búsqueda de información, es en este momento donde se sugiere que antes de iniciar un proceso de implementación a la industria 4.0 se motive a la capacitación de todas las áreas laborales de la empresa en materia tecnológica y la incursión, aunque lenta, segura para la empresa.

Para determinar el proceso de incursión, se ha estimado que es necesario mencionar el ejemplo de la empresa de estudio KRENDYC, la cual ha abordado de manera efectiva los avances tecnológicos pertinentes de la industria 4.0, es decir, esta empresa ha demostrado que ha buscado establecerse en catálogos digitales y en diversas plataformas de búsqueda, las cuales brindan información e interacción con los clientes, cuenta con 40 años en el ramo debido a su calidad y distinción de servicio sin embargo ha demostrado su facilidad de adaptación a los medios digitales actuales, así mismo, realiza sus procedimientos de distribución de manera eficaz y sobresaliente mediante diversas herramientas tecnológicas como rastreo, GPS, entre otras, así mismo ha implementado una gama de distribución y diversificación de sus productos, posicionándola como la empresa con mayor implementación 4.0.

En el informe "Perspectivas del sector industria" elaborado por la firma KPMG, en colaboración con la Confederación de Organizaciones Empresariales (CEOE), (KPMG; CEOE, 2018), estima que una de cada tres empresas no ha tomado medidas en relación a la Industria 4.0. De igual manera, al estudiar el caso de las empresas lácteas y su implementación tecnológica se determina que abordan el proceso de evolución digital realizando acciones aisladas, con la idea de que han realizado la implementación correcta al solo realizar la compra de tecnología, no consiguiendo el resultado esperado. 24

Mencionado lo anterior, se resaltan los resultados obtenidos mediante la aplicación y evaluación de las encuestas aplicadas, los cuales reflejan que las empresas lecheras evaluadas cuentan con un nivel bajo de implementación de avances tecnológicos correspondientes a la Industria 4.0. Sin embargo, dos de las empresas cuentan con avances tecnológicos adecuados para su competitividad regional, posicionándolas como las principales empresas distribuidoras en la región.

Las dos empresas que cuentan con avances tecnológicos en el área de distribución cuentan actualmente con capacidad de producción superior a la de sus competidores, una gama de productos diversa y con instrumentos de rastreo e interconexión en sus dispositivos y medios de transporte.

Al realizar una evaluación física de las empresas se destaca el poco personal que tienen en la fábrica de producción (De 8 a 10 personas), la maquinaria tiene pocos daños físicos y están interconectadas para reducir el tiempo de elaboración de sus productos, principalmente de los quesos que fabrican (Oaxaca, queso amarillo y canasto), los medios de transporte con los que laboran tienen integrado un mecanismo de rastreo, a su vez todo el personal está conectado mediante un dispositivo o aplicación virtual.

Contrariamente, las tres empresas que presentan baja implementación tecnológica, no presentan conocimiento de los dispositivos de rastreo modernos, tienen una idea cerrada de los procesos de distribución y desconocimiento de los términos abordados en las encuestas que se les presentó, demuestran una posición cerrada a la idea de modificar su empresa con tecnología debido a que su personal, o incluso los dueños, desconocen el uso de estos.

Al realizar un recorrido por las empresas y sus procesos de recolección de leche hasta la distribución, se puede visualizar el tamaño disminuido de sus plantas de fabricación, cuentan con gran personal (Mas de 15 personas) normalmente familiares, el $70 \%$ de las ventas son a pie de fábrica por lo que no consideran necesario equipar sus unidades (Principalmente carros de uso personal o bicicletas) con tecnología de punta.

Se concluye que las cinco empresas encuestadas consideran implementar en un futuro avances tecnológicos si el gobierno brinda un apoyo económico, así mismo, se identifica que el factor que los diferencia es el conocimiento y el poder adquisitivo que tienen cada uno, además de la apertura de conceptos en sus procesos.

Es por eso que se debe generar la idea de que estamos en una nueva realidad que exige de transformaciones directas a los procesos y elementos que incluyen las tecnologías habilitadoras tales como rastreo en línea, GPS, entre otros, que impactan en toda la cadena de valor y que al ser implementadas en cada fase conllevan a una apertura de oportunidades competitivas y diferenciadoras, esto con la finalidad de propiciar a las empresas a incursionar en la industria 4.0 como medio de competitividad y desarrollo económico, no solo sectorial sino a nivel estado.

Lo anterior, se debe a que en la actualidad estamos inmersos en un mercado de elevada incertidumbre, competitivo, complejo y de cambio acelerado, que implementa la tecnología como proceso estratégico y funcional para competir, por lo que las empresas lácteas 
en Tulancingo no pueden limitarse a esperar y ver qué resultados tiene el proceso de transformación digital en su competencia, si es exigida por el estado o incluso esperar a que su rezago ante las tecnologías y las necesidades de sus clientes se las exijan. Por tanto, resulta crucial que se acelere e inicie su transición a una Industria 4.0 de una forma colaborativa, buscando el apoyo de una compañía tecnológica de confianza que los incursione correctamente, y que las empresas busquen completar o realizar las claves que Álvarez proporciona, las cuales se mencionan a continuación.

(Álvarez, 2018), responsable de Transformación Digital e Industria 4.0 en la consultora tecnológica Izertis, miembro de la Comisión de Industria 4.0 de la Asociación Multisectorial de Empresas de Tecnologías de la Información, Comunicaciones y Electrónica (AMETIC), propone 9 cuestiones clave que toda empresa debe aplicar para incursionar de manera real en la industria 4.0 sin importar la fase de la cadena de valor con la que incursionen. 25

A continuación se enlistas en orden de importancia las cuestiones propuestas por Álvarez:

- Conocer qué beneficios e implicaciones tiene las tecnologías habilitadoras

- Abordar el proceso de transformación digital como una oportunidad y no como una amenaza.

- Definir una estrategia de transformación digital integral.

- Emprender el proceso de transformación de un modo sistémico.

- Diseñar una estrategia escalable.

- Desplegar una estrategia escalable.

- Construir una cultura empresarial abierta.

- Construir un plan de capacitación y gestión.

- Elaborar un plan de comunicación.

Se determina que las empresas lácteas evaluadas, caen constantemente en el error de no aplicar correcta y sistemáticamente los puntos anteriormente mencionados, debido a que, al realizar la visita a las empresas se pudo observar que a pesar de no contar con conocimientos y capital de inversión, han implementado diversas herramientas y equipo tecnológico dentro de sus áreas de trabajo, esto se interpreta como una implementación inconsciente, es decir, su necesidad laboral lo orilló a realizar la compra de tal herramienta tecnológica, sin embargo, no están implementando una industria 4.0 a sus procesos debido a que no emprenden un proceso de transformación sistémico y se estancan en esta incursión tecnológica.

Así mismo, al evaluar los resultados obtenidos en la encuesta aplicada a las empresas, se determina que el principal error de las empresas lecheras en la región de Tulancingo, Hidalgo, es creer que se puede implementar esta industria de manera rápida, comprando equipo tecnológico moderno y sin capacitación o inversión de capital a futuro. La implementación 4.0 es un procedimiento que se deriva en etapas y cumplimiento de metas, así como cumplimiento de objetivos. Para el caso de las empresas lácteas en Hidalgo, el procedimiento puede demorar más tiempo que en zonas con menor rezago tecnológico.

\section{Factores que entorpecen la implementación}

\section{de la industria 4.0 en las empresas lácteas de \\ Tulancingo}

Es fundamental impulsar la modernización del sector agroalimentario a través del apoyo a proyectos de inversión y capitalización que doten a las unidades productivas de maquinaria, equipo e infraestructura suficientes, que permitan elevar el nivel de productividad y rentabilidad y las hagan más competitivas en términos de rastreabilidad y sistemas de calidad.

Las características orográficas del territorio hidalguense, la falta de infraestructura y de servicios básicos, el grado promedio de escolaridad de la población de segundo de secundaria, y el uso de tecnologías obsoletas, son sólo algunos elementos que han limitado el desarrollo económico y social en la entidad.

En este apartado se presentan los resultados del cuestionario aplicado a las cinco empresas seleccionadas del sector lácteo de Tulancingo Hidalgo, las deducciones permiten la evaluación interna de los estándares tecnológicos con los que operan sus servicios de distribución. Se observa que el $40 \%$ de las empresas trabajan con avances tecnológicos en sus procesos de transporte, además de manejar dispositivos de rastreo competente, sin embargo, el $60 \%$ de las empresas evaluadas no han logrado implementar la tecnología necesaria para ser competitivas a nivel estatal.

Se ha determinado que, a nivel estado, existe una diversidad de factores que influencian la baja implementación de la industria 4.0, uno de los factores externos, es el insuficiente apoyo gubernamental para la innovación, capacitación, e investigación en el área de tecnología; así como la falta de concientización y de información a los empresarios respecto a la industria 4.0. Así mismo, para determinar los factores externos que influencian en la implementación de la industria 4.o dentro de las empresas lácteas en la región de Tulancingo, Hidalgo, se debe determinar, "En primera instancia, que dentro de la cadena productiva de leche de vaca en el Estado de Hidalgo se presentan seis eslabones: proveedores de insumos, sistemas de producción, acopio, transformación, distribución y consumidor final", (Cuevas Reyes, 2007). 26

El eslabón en el que este trabajo se enfoca es el de distribución, el cual incluyó la caracterización de los 
distribuidores mayorista, minorista y detallista de productos lácteos y, por lo tanto, es el eslabón que presenta mayor índice de rezago tecnológico en estas industrias.

De igual manera, se identifican algunos de los factores críticos de la cadena agroalimentaria que afectan de forma directa la competitividad de la cadena de distribución de leche en el Estado de Hidalgo, factores resultantes del trabajo de investigación titulado "Technological and policy demands to improve the milk agro-alimentary chain in Hidalgo State", (Romero Santillán, et al., 2009). 27

Dichos problemas se presentan a continuación:

- Altos costos de implementación tecnológica.

- Deficiente manejo y almacenamiento del producto.

- Deficientes esquemas de transferencia de tecnología en los sistemas de producción y pequeñas agroindustrias.

- Existe una deficiente organización de los productores con tecnología intermedia y baja.

- Limitada infraestructura en los sistemas de producción y agroindustrias.

Así mismo, según un estudio realizado por Cuevas, (Cuevas Reyes, 2007), en consulta con un foro de diversos agentes de la cadena, establecen que los factores críticos de mayor importancia que limitan la competitividad de la cadena agroalimentaria de leche en Hidalgo. 28

Dichos factores críticos se presentan a continuación:

- Deficiente organización de los productores con tecnología intermedia y baja.

- Limitada infraestructura en los sistemas de producción y agroindustrias pequeñas de la cadena.

- Deficientes esquemas de transferencia de tecnología en los sistemas de producción y pequeñas agroindustrias.

Mencionando lo anterior, se realizó un análisis de los factores internos que afectan directamente a las empresas evaluadas, por lo que, mediante la visita a sus instalaciones se determinaron los factores internos que retrasan o, en dado caso, entorpecen la implementación de la industria 4.0 a sus procesos de distribución dentro de las empresas lácteas en la región de Tulancingo, esto podría interpretarse como una de las razones por las cuales estas empresas no han desarrollado las habilidades pertinentes para incursionar en lo que conocemos como una empresa 4.0, por lo tanto, en la industria 4.0.

Los factores internos que a continuación se presentan en forma enlistada, se obtuvieron mediante la visualización del equipo de trabajo, el personal, los procesos y las carencias notorias que presentan las empresas evaluadas en función de optimización y cumplimiento de metas para la implementación de la industria 4.0:

- Rezago en el uso e implementación de Tecnologías de la Información y Comunicación (TIC's)

- Deficiente flexibilidad y capacidad de adaptación de tecnologías.

- Carencia de medios de financiamiento: para personal y equipo de trabajo.

- Bajo nivel educativo del personal.

- Limitado recurso de entrenamiento y capacitación tecnológica.

- Dependencia de redes existentes.

- Deficiente visión y motivación empresarial.

- Deficiente capacidad de innovación.

- Rezago en materia de tecnología.

Por lo tanto, se puede interpretar que las empresas lácteas presentan factores externos que retrasan la implementación de la industria $4.0 \mathrm{y}$, el acceso a conocimientos en materia tecnológica y, factores internos que entorpecen el procedimiento de incursión de esta industria.

Identificando los factores externos que impiden o retrasan la implementación de la industria 4.0 se debe visualizar la posición en la que se encuentra el Estado de Hidalgo ante la implementación y desarrollo en ciencia, tecnología e innovación (CTI), debido a que éstos son factores clave para acelerar el crecimiento económico, la competitividad internacional, la sustentabilidad ambiental y las mejoras en el bienestar de una nación, debido a que se implementan con la finalidad de destinar recursos públicos, promover la participación de las empresas privadas en el financiamiento de las actividades de investigación e innovación.

El artículo "Estímulos fiscales para promover la inversión en ciencia, tecnología e innovación”, publicado en el Núm. 33 de la revista Pluralidad y Consenso del Instituto Belisario Domínguez, (Amador Hernández, 2017), revela que, en las últimas tres décadas la inversión privada ha disminuido sistemáticamente hasta llegar a una participación menor al 20\% en el Gasto en Investigación y Desarrollo Experimental (GIDE), que es el indicador utilizado internacionalmente para medir la inversión en ciencia y tecnología. En contraparte, la inversión pública en $\mathrm{CTI}$ se ha duplicado, con una participación en los últimos años de alrededor del $70 \%$ en el GIDE. 29

La nota informativa publicada por la Oficina de Información Científica y Tecnológica para el Congreso de la Unión (INCyTu) (Oficina de Información Científica y Tecnológica para el Congreso de la Unión, 2018), establece que, los países desarrollados dedican entre $1.5 \%$ y $4.2 \%$ de su PIB al GIDE. En México el valor de este indicador se ha quedado rezagado durante décadas sin rebasar el $0.55 \%$, valor alcanzado en el año 2015. 30 
De acuerdo al estudio realizado por el Foro Económico Mundial, Foro creado por el economista alemán Klaus Schwab en 1971, menciona que el $88 \%$ de las organizaciones aún no entienden las implicaciones de la Industria 4.0 para sus modelos de negocio, por lo que los empresarios la consideran como innecesaria y con poca importancia, (WeFORUM (Schwab, K.), 2017). 31

Por lo tanto, al analizar los resultados presentados en las encuestas de evaluación, se puede dar respuesta a la pregunta planteada inicialmente, ¿Las empresas que no han logrado implementar la industria 4.0, realmente son incapaces de adoptar el desarrollo derivado de esta nueva revolución? ó ¿Existe un factor externo que les impide realizar dichos cambios?

$\mathrm{Si}$ bien se ha demostrado mediante los resultados obtenidos por las empresas evaluadas que existen factores que entorpecen el proceso de industrialización 4.0 , no representan un obstáculo que incapacite o anule dicho proceso, sin embargo, se demostró que aquellas empresas que no han incursionado en la industria 4.0 tienen como principal factor la falta de conocimiento y, por lo tanto, estímulos para incursionar, a su vez, se puede concluir que si bien tienen factores (internos y externos) que entorpecen su implementación tecnológica, dichas empresas no son incapaces de incursionar en la industria 4.0.

Con la finalidad de dar sustento, se presenta a continuación la Tabla 1, la cual presenta las posiciones en las que los estados se ubican de acuerdo al nivel de inversión que dedican a las áreas de ciencia, tecnología e innovación, con datos del Ranking Nacional de Ciencia, Tecnología e Innovación para los años 2015 a 2018, publicados en el Foro Consultivo, (Foro Consultivo Científico y Tecnológico, 2018). 32

En la tabla que se presenta a continuación, observa que el estado de Hidalgo presenta un nivel de emprendimiento de alto rendimiento posicionándolo en el $8^{\circ}$ lugar, así mismo se posiciona en el lugar 14 dentro del Ranking para los sectores de Inversión Pública y Privada en CTI lo cual refleja que cuenta con un nivel competitivo aunque no tan favorable en comparación con el nivel de emprendimiento y negocios que se desarrollan dentro del Estado, para el caso de los sectores de Producción Científica y Propiedad Industrial, el Estado de Hidalgo se encuentra en la 19 posición lo cual demuestra que el Estado requiere de especialización y protección de inventos, así mismo el Estado de Hidalgo presenta un ligero rezago para los sectores de Empresas Innovadoras y Tecnologías de la Información, en la 21 posición del Ranking.

Así mismo, observa que el estado de Hidalgo presenta un nivel de emprendimiento de alto rendimiento posicionándolo en el $8^{\circ}$ lugar, así mismo se posiciona en el lugar 14 dentro del Ranking para los sectores de
Inversión Pública y Privada en CTI lo cual refleja que cuenta con un nivel competitivo aunque no tan favorable en comparación con el nivel de emprendimiento y negocios que se desarrollan dentro del Estado.

\begin{tabular}{|c|c|c|c|c|c|c|c|}
\hline $\begin{array}{l}\text { Entidad } \\
\text { Federativa }\end{array}$ & \begin{tabular}{|c|} 
Invers \\
ión \\
Públic \\
a- \\
Privad \\
a en \\
CTI \\
\end{tabular} & $\begin{array}{l}\text { Produc } \\
\text { ción } \\
\text { Científi } \\
\text { ca }\end{array}$ & $\begin{array}{c}\text { Empres } \\
\text { as } \\
\text { Innovad } \\
\text { oras }\end{array}$ & $\begin{array}{l}\text { Emprendim } \\
\text { iento y } \\
\text { Negocios }\end{array}$ & $\begin{array}{c}\text { Infraestru } \\
\text { ctura } \\
\text { Material e } \\
\text { Intelectual }\end{array}$ & $\begin{array}{c}\text { Propie } \\
\text { dad } \\
\text { Industr } \\
\text { ial }\end{array}$ & $\begin{array}{c}\text { Tecnolo } \\
\text { gías de } \\
\text { la } \\
\text { Informa } \\
\text { ción }\end{array}$ \\
\hline $\begin{array}{l}\text { Aguascali } \\
\text { entes }\end{array}$ & 17 & 18 & 10 & 7 & 16 & 8 & 7 \\
\hline $\begin{array}{l}\text { Baja } \\
\text { California }\end{array}$ & 8 & 6 & 32 & 28 & 20 & 12 & 2 \\
\hline $\begin{array}{l}\text { Baja } \\
\text { California } \\
\text { Sur }\end{array}$ & 4 & 8 & 13 & 3 & 15 & 21 & 6 \\
\hline Campeche & 30 & 16 & 19 & 4 & 7 & 15 & 17 \\
\hline Coahuila & 11 & 13 & 7 & 20 & 8 & 7 & 16 \\
\hline Colima & 7 & 11 & 14 & 1 & 3 & 20 & 9 \\
\hline Chiapas & 31 & 29 & 2 & 15 & 26 & 31 & 32 \\
\hline Chihuahua & 10 & 17 & 30 & 22 & 19 & 16 & 8 \\
\hline \begin{tabular}{|l|} 
Ciudad de \\
México
\end{tabular} & 1 & 1 & 1 & 32 & 1 & 1 & 1 \\
\hline Durango & 22 & 23 & 15 & 10 & 5 & 27 & 20 \\
\hline $\begin{array}{l}\text { Guanajuat } \\
0\end{array}$ & 20 & 12 & 16 & 24 & 22 & 5 & 25 \\
\hline Guerrero & 27 & 32 & 18 & 9 & 28 & 32 & 30 \\
\hline Hidalgo & 14 & 19 & 21 & 8 & 14 & 19 & 21 \\
\hline Jalisco & 18 & 14 & 3 & 18 & 27 & 3 & 12 \\
\hline México & 19 & 24 & 17 & 25 & 31 & 17 & 10 \\
\hline Michoacán & 21 & 15 & 29 & 27 & 18 & 22 & 28 \\
\hline Morelos & 2 & 2 & 24 & 17 & 2 & 6 & 13 \\
\hline Nayarit & 23 & 25 & 25 & 5 & 25 & 26 & 19 \\
\hline $\begin{array}{l}\text { Nuevo } \\
\text { León }\end{array}$ & 12 & 4 & 5 & 23 & 12 & 2 & 3 \\
\hline Oaxaca & 25 & 31 & 20 & 31 & 32 & 30 & 31 \\
\hline Puebla & 16 & 10 & 12 & 14 & 6 & 10 & 26 \\
\hline Querétaro & 3 & 3 & 11 & 16 & 11 & 4 & 5 \\
\hline $\begin{array}{l}\text { Quintana } \\
\text { Roo }\end{array}$ & 28 & 28 & 23 & 6 & 29 & 11 & 11 \\
\hline $\begin{array}{l}\text { San Luis } \\
\text { Potosí }\end{array}$ & 6 & 7 & 27 & 29 & 24 & 24 & 24 \\
\hline Sinaloa & 13 & 20 & 4 & 2 & 23 & 14 & 14 \\
\hline Sonora & 15 & 9 & 9 & 12 & 10 & 13 & 4 \\
\hline Tabasco & 32 & 27 & 8 & 19 & 30 & 23 & 22 \\
\hline $\begin{array}{l}\text { Tamaulipa } \\
\text { s }\end{array}$ & 26 & 30 & 31 & 13 & 17 & 18 & 15 \\
\hline Tlaxcala & 9 & 26 & 28 & 21 & 21 & 28 & 23 \\
\hline Veracruz & 24 & 22 & 26 & 30 & 13 & 29 & 27 \\
\hline Yucatán & 5 & 5 & 6 & 11 & 4 & 9 & 18 \\
\hline Zacatecas & 29 & 21 & 22 & 26 & 9 & 25 & 29 \\
\hline
\end{tabular}

Tabla 1. Ranking Nacional de Ciencia, Tecnología e

Innovación (2015 -2018).

\section{Fuente: Elaboración propia con datos de Ranking}

Nacional de Ciencia, Tecnología e Innovación, (2020).

En la tabla anterior se puede observar que los sectores de Producción Científica y Propiedad Industrial, el Estado de Hidalgo se encuentra en la 19 posición lo cual demuestra que el Estado requiere de especialización y 
protección de inventos, así mismo el Estado de Hidalgo presenta un ligero rezago para los sectores de Empresas Innovadoras y Tecnologías de la Información, en la 21 posición del Ranking.

De igual manera, refleja la importancia de la industrialización de las empresas, así como la implementación de avances tecnológicos en sus procesos, así mismo, podemos observar que los fondos económicos y el apoyo del gobierno son factores críticos para el desarrollo de las industrias y empresas dentro de los rubros evaluados. Se visualiza al Estado de Hidalgo en un nivel medio, lo cual se podría interpretar como un Estado competente, sin embargo, requiere aumentar la innovación como medida de competitividad.

Por lo anterior, podemos concluir que los factores primordiales que debe afrontar una empresa que busca la implementación de la industria 4.0 son los internos, debido a que, en la experiencia laboral que comparten las empresas evaluadas, se ha demostrado que el proceso de implementación tecnológica es más sencillo, aunque igual de costoso, para aquellas empresas que siguen un modelo escalable y sistémico, con metas y objetivos establecidos de manera razonable y realista, es por esta razón que los factores internos se posicionan como primordiales. No obstante, los factores externos que se presentaron en este apartado, son de vital importancia, debido a que, si bien la empresa no tiene control sobre ellos, el conocimiento sobre estos factores permitiría la búsqueda de alternativas 0 incluso, el tomar precauciones a estos.

El nivel con que el Estado de Hidalgo se posiciona en materia de competitividad, emprendimiento e innovación, tiene como finalidad demostrar que es un Estado competente, que permite el desarrollo y crecimiento de las empresas, aunque bien no es un impulsor al desarrollo tecnológico se considera como un factor externo con posibilidades de superación.

\section{Implementación de la industria 4.0 a los procesos de distribución, estrategia de competitividad}

"Hay tres razones por las que las transformaciones actuales no representan una prolongación de la tercera revolución industrial, sino la llegada de una distinta, la cuarta revolución industrial: la velocidad, el alcance y el impacto en los sistemas. "La velocidad de los avances actuales no tiene precedentes en la historia... Y está interfiriendo en casi todas las industrias de todos los países", apunta el Foro Económico Mundial (WEF) por sus siglas en inglés, (WeFORUM (Schwab, K.), 2017). 33 Por lo anterior, la opción para las industrias lácteas y su competitividad es la implementación de los nuevos avances tecnológicos que integran a la industria 4.0; este es el mejor camino en un mundo globalizado, caracterizado por economías integradas y mercados competitivos y, la industria lechera no es la excepción. "En este contexto, se configura un escenario donde la competencia generalizada exige la implementación de estrategias para mejorar permanentemente la productividad y rentabilidad en el sector lechero, siendo la base para garantizar la permanencia competitiva de las empresas lecheras en el mercado, por méritos propios", (Loera \& Banda, 2017). 34

Joachim Miebach, socio y fundador de Miebach Consulting, en su artículo publicado en la revista Énfasis Logística "Industria 4.0: desafíos para los procesos logísticos y la automatización de la manipulación de materiales", (Miebach \& Ribas, 2017), señaló que las áreas de almacenes y distribución están revolucionando los procesos operativos debido a la integración de la industria 4.0 y al nivel de optimización, organización y control que ejerce ésta industria, así mismo los procesos de distribución son en tiempo real, brinda entregas en tiempos más cortos y una masificación de la producción,. Más allá de los procesos de diseño y producción de bienes, las tecnologías de la Industria 4.0 pueden impactar también en la forma en que los productos terminados se mueven en el entorno, se almacenan y se distribuyen, se ha observado un aumento de las tecnologías conectadas a la cadena de valor de fabricación y distribución. 35

Esta unión de sistemas digitales y físicos ha permitido que se construyan plataformas que brindan experiencias cada vez más conectadas que impactan desde el diseño y la planificación de los productos, la cadena de suministro y la producción de bienes. Sin embargo, las tecnologías inherentes a la industria 4.0 también pueden afectar la manera del acabado de los productos, la manera en que se almacenan y se distribuyen, (Taliaferro, et al., 2018). 36

Las tecnologías y avances de la industria 4.0 que requieren las empresas lácteas deben ser seleccionadas correctamente conforme al canal utilizado y, por lo tanto, la tecnología que éste requiere, es decir, el beneficio de lugar y de tiempo que éste presentará para los procesos y capacidades con que cuentan las empresas lácteas. Es decir, el beneficio de lugar se refiere a la distancia recorrida por el vendedor y el consumidor para concluir con el proceso de la compra-venta y, el beneficio de tiempo hace referencia a que el producto sea llevado en el momento adecuado y con mayor rapidez.

Mencionado lo anterior, se enlistan los tipos de distribución y los requerimientos tecnológicos requeridos:

- Distribución de los productos directamente a los consumidores.

- Distribución a minoristas

- Distribución a supermercados. 
- Distribución a los mayoristas.

Sin embargo, aunque la implementación de la industria 4.0 es un paso fundamental para la productividad, la rentabilidad y la competitividad de estas empresas lácteas, no es todo lo que pueden y deben hacer los productores lecheros, independientemente de lo que ocurra o deje de ocurrir en el ámbito tecnológico e industrial.

\section{Resultados de las encuestas aplicadas}

A lo largo de este trabajo se mencionaron los diversos resultados obtenidos en las encuestas aplicadas a las cinco empresas lácteas de investigación, sin embargo, en este apartado se pretende dar análisis a los resultados que se obtuvieron.

Para efectos de un mejor análisis, las preguntas se presentarán en dos secciones: la primera de acuerdo a su grado de implementación de avances tecnológicos pertinentes a la industria 4.0 en los procesos de distribución, la segunda de acuerdo a la implementación de diversos elementos tecnológicos en las empresas, así como el análisis de sus conocimientos, para esto se presentarán mediante una gráfica de pastel que permita visualizar los resultados obtenidos en la aplicación de las encuestas, a su vez, en la parte inferior de los gráficos se podrá observar el análisis del resultado dentro de este trabajo.

Mencionado la anterior, se presenta a continuación la Gráfica 1, correspondiente a la tercer pregunta del cuestionario aplicado, es en esta donde se puede observar que, 2 de las empresas evaluadas tienen una distribución rígida (cerrada) donde transportan pocas piezas, contrariamente, 3 empresas han desarrollado una distribución flexible a las necesidades de sus clientes, esto se interpreta como una posibilidad de adaptación a las necesidades vanguardistas de los clientes $y$, por lo tanto, la posibilidad de inversión futura en innovaciones tecnológicas.

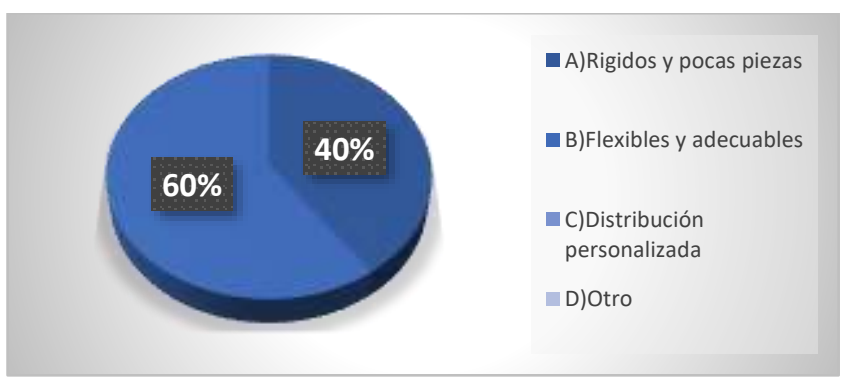

Gráfica 1. Distribución de lotes pequeños

Fuente: Elaboración Propia con datos de encuesta.

La Gráfica 2, que se presenta a continuación, representa a continuación, demuestra el rezago en avances tecnológicos implementados a las unidades de distribución en las empresas lácteas evaluadas debido a que el $60 \%$ de las empresas aseguran desconocer los servicios de $\mathrm{TI}$ aplicables a la distribución de su producto, a su vez, mencionan desconocer los términos y los centros de implementación de dicha tecnología, contrariamente a esto, tan solo $20 \%$ de las empresas han implementado estos servicios mediante aplicaciones y, una de las empresas está en proceso de implementar los Servicios $\mathrm{Tl}$ a sus unidades de distribución, sin embargo, al analizar los resultados se estipula que las empresas que aseguran contar con este servicio desconocían en primer instancia los términos y, por lo tanto, desconocían que contaban con dichos servicios.

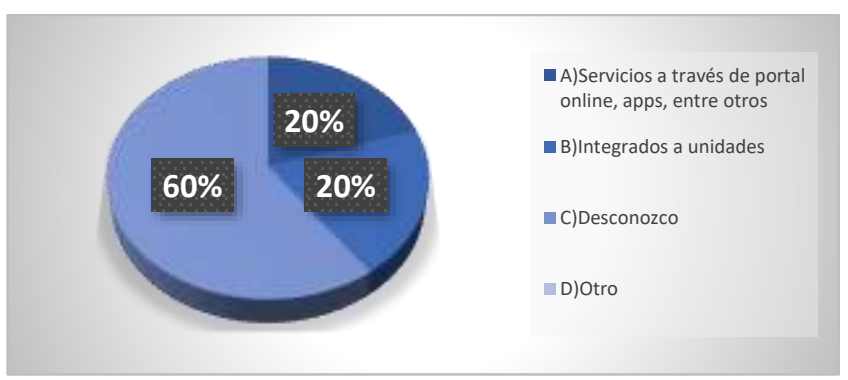

Gráfica 2. Servicios de TI asociados a la distribución

Fuente: Elaboración Propia con datos de encuesta.

De igual manera, se ha comprobado mediante la Gráfica 3 , que 4 de las empresas evaluadas no cuentan con un monitoreo de unidades debido a que consideran que su producto no requiere de cuidados o supervisión en el proceso de distribución, sin embargo, $40 \%$ de estas aseguran que realizan un monitoreo de su personal por medio de un monitoreo básico, es decir, por llamadas telefónicas. A su vez, tan solo 1 empresa evaluada asegura que rastrea sus unidades mediante el uso de aplicaciones, debido a que se distribución es a diversas localidades y sus transportistas requieren conocer las rutas para completar su entrega.

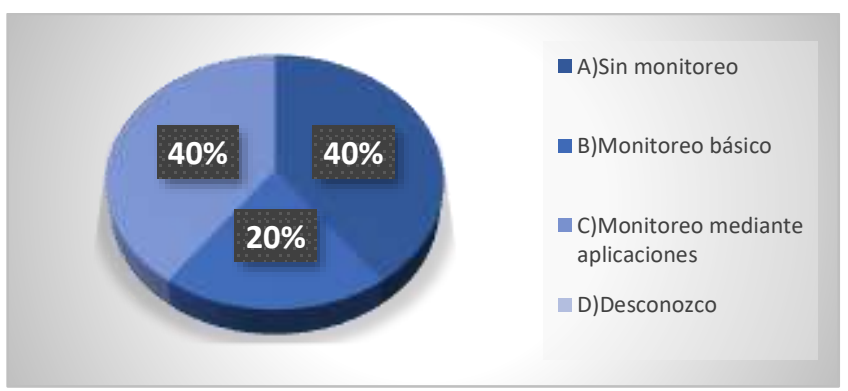

Gráfica 3. Monitoreo de unidad/ producto

Fuente: Elaboración Propia con datos de encuesta.

A continuación, se presenta la Gráfica 4, que tiene como finalidad evaluar la infraestructura TIC de las empresas evaluadas, esta infraestructura se refiere a todas las 
tecnologías que interfieren y gestionan los procesos informativos y de comunicación de personas y máquinas, es decir, engloba los mercados de hardware, software, telecomunicaciones, automatización y comunicación de negocios y servicios de TI.

Es aquí donde se observa que el $80 \%$ de las empresas evaluadas no han incursionado en la industria 4.0, debido a que no cuentan con ninguno de los apartados que integra esta infraestructura, mencionados anteriormente $y$, la empresa que asegura contar con la infraestructura TIC, usa datos inalámbricos para imprimir ordenes de productos, así como para la comunicación con sus transportistas, sin embargo no clasifica dentro de dicha implementación, aunque se considera una empresa con potencial incursión.

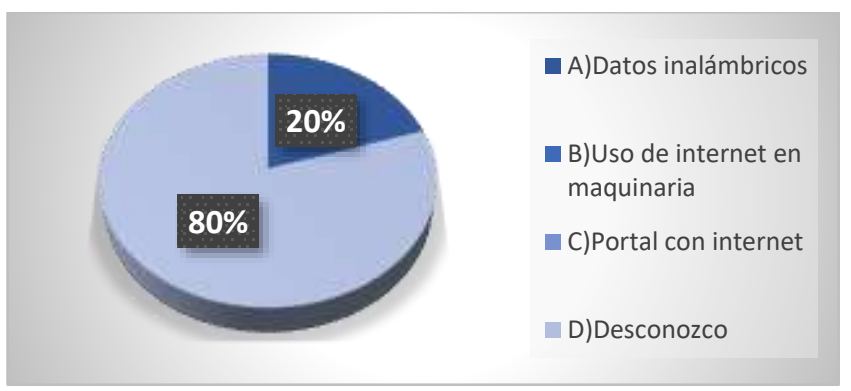

Gráfica 4. Infraestructura TIC

\section{Fuente: Elaboración Propia con datos de encuesta.}

Con la finalidad de evaluar los medios que se utilizan para realizar la conexión entre áreas o departamentos dentro de las empresas evaluadas, se presenta a continuación la Gráfica 5, la cual tiene como opciones una comunicación que implementa avances tecnológicos, conexiones directas (persona a persona) o en caso contrario, no cuenten con conexiones o departamentos alternos. La evaluación demostró que las empresas evaluadas no cuentan con ningún avance tecnológico debido a la infraestructura con que cuentan, es decir, todas las áreas de la empresa se encuentran dentro de la misma zona y laboran mediante una conexión básica, es decir, persona a persona y, al menos dos empresas no cuentan con ningún tipo de conexión entre sus áreas.

Los gráficos anteriores muestran los resultados obtenidos mediante la aplicación de la encuesta previamente presentada, es en este punto donde se observa el desconocimiento de las diversas tecnologías aplicables a sus medios de transporte dentro de las empresas lácteas, sin embargo, una de las empresas demostró un avance en materia de tecnología al implementar sensores en sus unidades y, dentro de sus instalaciones físicas, esto como medida de seguridad, sin embargo, no son equipos de alta tecnología, a su vez, las empresas reportan desconocer los servicios de $\mathrm{Tl}$ asociados a los procesos de distribución, es en este punto donde se recalca la necesidad de capacitar e informar a las empresas las nuevas necesidades tecnológicas del mercado.

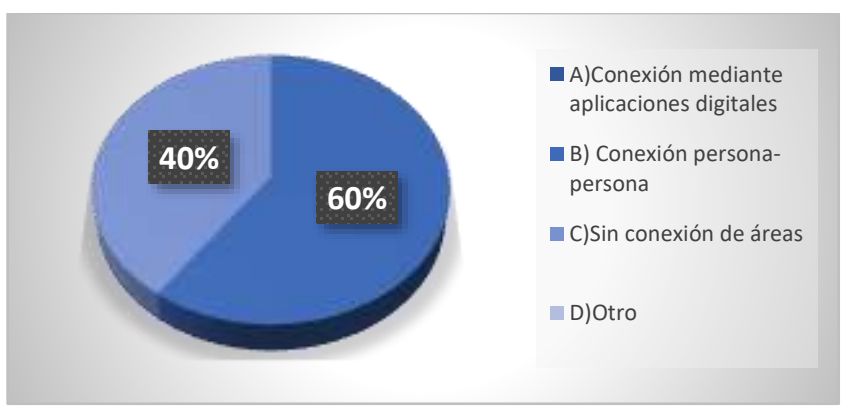

Gráfica 5. Conexión de áreas- departamentos

\section{Fuente: Elaboración Propia con datos de encuesta.}

De igual manera se pudo observer el rezago tecnológico dentro de las empresas seleccionadas, debido a que, el $100 \%$ de las empresas reportan no tener un almacén de datos en la nube o mediante el uso de medios tecnológicos, es en este punto donde se visualiza que las empresas seleccionadas consideran de poca importancia el almacenar los datos obtenidos de sus ventas, compras, proveedores, entre otros, y, tan solo el $40 \%$ de las empresas evaluadas reportaron almacenar sus datos en papel, por lo que no se completa un proceso de intercambio de datos digital, esto ultimo, es un tema de discusión dentro de este trabajo, debido a que, como se mencionó en apartados anteriores, la capacidad de producción en el $60 \%$ de las empresas no reclama de estas actividades y por lo tanto, las empresas lo consideran de poca necesidad e importancia.

A su vez, tan solo $20 \%$ de las empresas consideran que un modelo de negocios que implemente las novedades tecnológicas y los incursione en esta industria es importante, y estan en disposición de implementarlo, se considera que el $80 \%$ de las empresas restantes no consideran esta opción debido al costo de inversion requerido y el desconocimiento de lo que conlleva una implementación tecnológica de este nivel.

Es por esta razón que se presentan a continuación lo resultados de las preguntas cuyo propósito es ilustrar el nivel en que la empresa ha incursionado en la industria 4.0, para conocer el nivel de incursión se decidió evaluar las preguntas que reflejen el conocimiento que las empresas tienen de las tecnologías actuales, a su vez, conocer si se han implementado interfaces personamáquina-máquina, en caso de contar con estos, la empresa tendría un avance tecnológico que le proporcionaría competitividad no solo a nivel regional.

Con la finalidad de evaluar los dispositivos tecnológicos implementados dentro de las empresas evaluadas, se presenta a continuación la Gráfica 6, es en esta donde se ilustran los resultados obtenidos en la primer pregunta del 
cuestionario aplicado, es decir, $80 \%$ las empresas aseguran no contar con sensores dentro de sus unidades de distribución o sus instalaciones físicas, debido a que no tienen conocimiento del uso de los sensores y consideran que para el giro comercial al que pertenecen, no es necesario implementarlos, contrariamente a esto, una empresa asegura que cuenta con sensores dentro de sus instalaciones y sensores de puerta en su zona de carga.

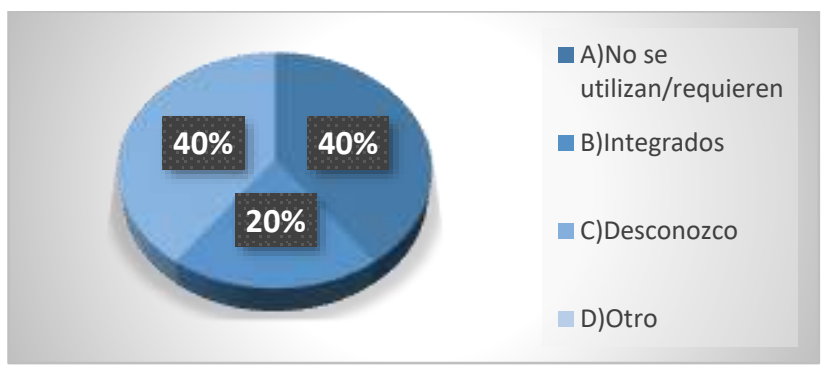

Gráfica 6. Integración de Sensores

Fuente: Elaboración Propia con datos de encuesta.

A su vez, se evaluó el medio con que las empresas realizan el intercambio de datos entre los departamentos o áreas de la empresa, la Gráfica 7 ilustra los resultados obtenidos en este rubro. Podemos observar que, el $60 \%$ de las empresas evaluadas no realizan almacenaje de datos debido a las funciones que estas desempeñan y, el desconocimiento de la importancia de almacenar, procesar e intercambiar datos entre sus departamentos o para realizar un estudio del potencial para mejorar la empresa.

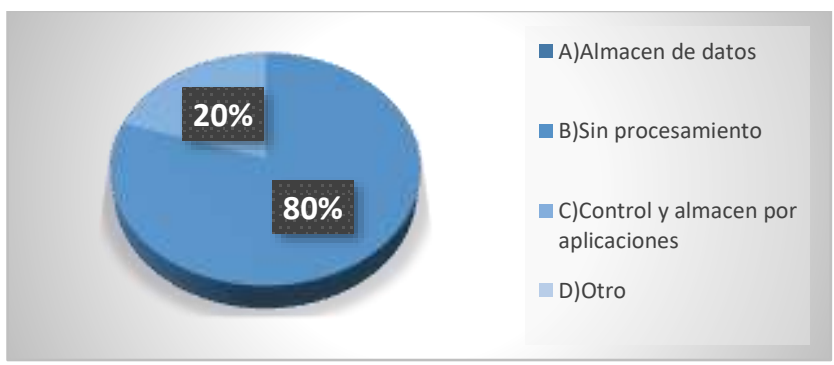

Gráfica 7. Procesamiento de datos

Fuente: Elaboración Propia con datos de encuesta.

Como se mencionó anteriormente, las empresas evaluadas no cuentan con actividades o dispositivos de almacenaje de datos, esto se debe principalmente al desconocimiento, sin embargo, se ha comprobado mediante la aplicación de este cuestionario que los empresarios subestiman la importancia que estos procesos conllevan dentro de las empresas. Mediante la evaluación de los resultados, se ha llegado a la conclusión que las empresas evaluadas tienen un rezago significativo en materia de tecnología y, tan solo $20 \%$ de las empresas lácteas evaluadas de esta región han creado un estado de conciencia sobre las ventajas que representa el uso de tecnologías en sus procesos, por lo que no se aprecia implementación de la industria 4.0 en este sector.

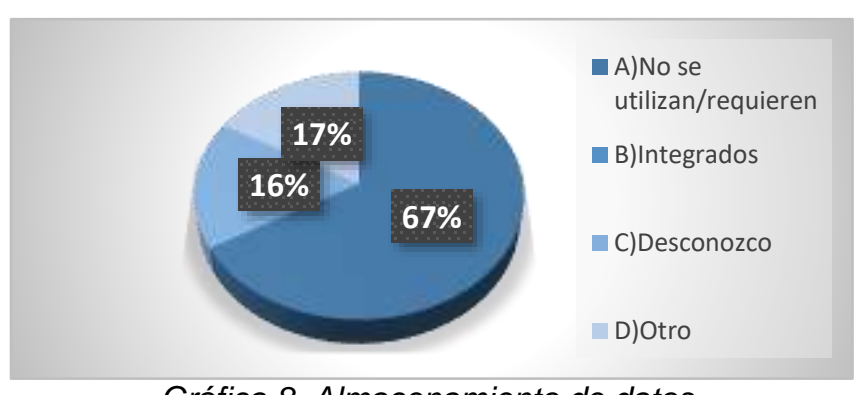

Gráfica 8. Almacenamiento de datos

\section{Fuente: Elaboración Propia con datos de encuesta.}

Mediante la Gráfica 9, se presentan los resultados obtenidos en la segunda pregunta del cuestionario aplicado, la cual refleja que el $60 \%$ de las empresas no cuentan con un intercambio de datos entre sus áreas o departamentos debido a que no realizan funciones de recopilación o procesamiento de datos por lo que no han establecido funciones para este apartado, sin embargo, 2 de las empresas evaluadas aseguran intercambiar datos con sus distribuidores y transportistas debido a que estos requieren la información de clientes así como aquellos datos necesarios para completar los procesos de distribución y venta.

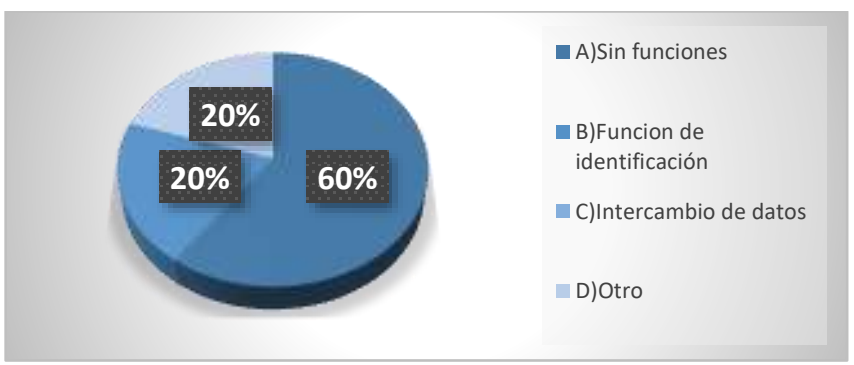

Gráfica 9. Intercambio de Datos

Fuente: Elaboración Propia con datos de encuesta.

Como se mencionó anteriormente, las empresas evaluadas aseguran compartir información y datos entre su personal de ventas y distribución, es por esta razón que mediante la gráfica 10 , se ilustra que el $40 \%$ de las empresas realizan esta comunicación de persona a persona, el otro $40 \%$ de las empresas asegurar utilizar dispositivos electrónicos y aplicaciones móviles para el contacto entre el personal, sin embargo, una empresa asegura no contar con ningún medio de comunicación o conectividad debido a que sus departamentos no 
comparten actividades que requieran la comunicación entre ellas.

De igual manera, se evaluaron los avances y interconexiones implementadas en los procesos internos de las empresas, obteniendo como resultado un scenario poco favorable para la industria 4.0. Los resultados se muestran a continuación.

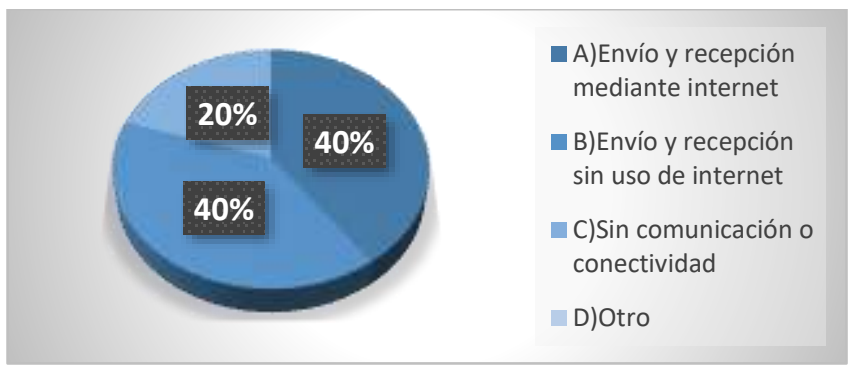

Gráfica 10. Comunicación- Conectividad

Fuente: Elaboración Propia con datos de encuesta.

Una zona de comunicación entre el personal y la maquina con que se trabaja es de vital importancia debido a que esta tiene como objetivo permitir el funcionamiento y control más efectivo de la máquina desde la interacción con el humano, sin embargo, las empresas que se evaluaron para fines de estudio, han demostrado que no cuentan con una interfaz entre persona- maquina, debido a que su infraestructura $\mathrm{TI}$ es nula, es decir, su maquinaria no está integrada con tecnología. Sin embargo, una empresa asegura contar con un interfaz de este tipo, debido a que utiliza dispositivos inteligentes, un área administrativa que utiliza internet y equipo tecnológico. (Ver Gráfica 11)

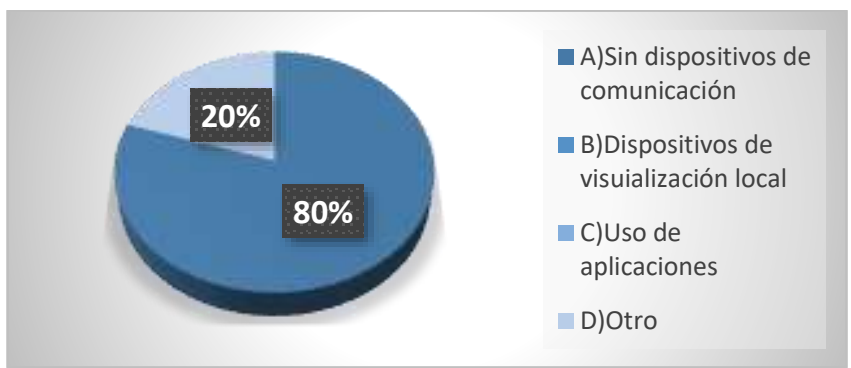

Gráfica 11. Interfaz persona-máquina

\section{Fuente: Elaboración Propia con datos de encuesta.}

Como se mencionó anteriormente, las empresas no cuentan con interfaces entre persona-máquina por lo que la comunicación entre su maquinaria es inexistente (Ver Gráfica 12), esto es comprensible debido a que las empresas no han implementado maquinaria inteligente a sus procesos, posiblemente por el alto costo que requiere esta implementación, no solo por la maquinaria, sino porque es necesario adaptar una infraestructura $\mathrm{TI}$ a la empresa y, como se ha mencionado a lo largo de este trabajo, las empresas no cuentan con el capital o la capacitación permitente para estos avances tecnológicos, esto es un tema de valoración debido a que este sector ha demostrado que la implementación de la industria 4.0 a sus procesos es de lenta acción, la zona donde se desarrolle la empresa y, sobre todo, la competencia a la que se enfrente son los factores próximos a evaluar antes de implementar un avance tecnológico escalable.

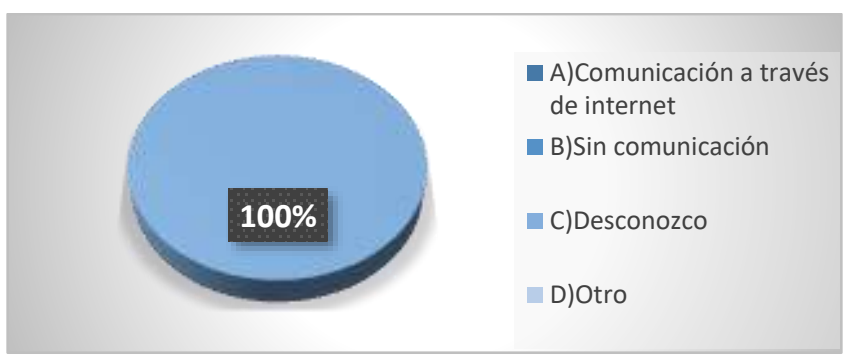

Gráfica 12. Comunicación máquina-máquina

Fuente: Elaboración Propia con datos de encuesta.

Se resalta que el $40 \%$ de las empresas evaluadas cuentan con oportunidades debido a su manejo de medios electrónicos, así mismo, se considera que su implementación tecnológica se debe a los años de experiencia en el mercado, es decir, es una empresa que ha sido innovadora en sus procesos por lo que ha perdurado en compararción a su competencia, sin embargo, es necesario impulsar a toda empresa a desarrollar una infraestructura TIC como medida de competitividad. Los resultados en este apartado demuestran que las empresas lácteas en esta region tienen un alto índice de desventaja, por lo que integrar avances tecnológicos en su departamento de distribución se considera necesario, esto solo en función de su capacidad de distribución y un modelo de negocios que los integre de manera escalable y personalizada en la industria 4.0 .

\section{Retos y oportunidades que presentan las empresas lácteas en Tulancingo ante la implementación de la industria 4.0 en sus procesos de distribución}

En este trabajo se ha mencionado la importancia de implementar estas tecnologías a los procesos de distribución debido a las grandes ventajas que representa para estas industrias ante su competencia, sin embargo, es necesario conocer los retos y oportunidades a los que se enfrentan dichas empresas, con el fin de comprender el porqué del rezago tecnológico en el Estado de Hidalgo. 
Para profundizar en los puntos principales que reflejaron un reto para las empresas lácteas, podemos mencionar a Klaus Schwab, quien en su libro titulado "La cuarta revolución industrial", asegura que los desafíos creados por esta industria radican en el lado del proveedor, en el mundo del trabajo y la producción debido a la caída del precio relativo de los bienes de inversión, impulsados a la vez por el progreso de la innovación.

Se puede interpretar lo anterior como el nivel de intervención que tiene la innovación sobre el uso de capital y trabajo, obligando a las empresas que buscan la implementación de tecnologías de punta, así como automatización de sus procesos en conjunto con los avances que representa esta industria a sustituir el nivel de trabajo por un nivel de capital mayor.

Es en este punto donde surge el segundo reto para estas empresas lácteas, el capital humano, como mencionan en el reporte Workforce of the Future, (Deloitte Touche Tohmatsu Limited, 2017), es importante invertir en la educación, la transformación digital, la automatización, el manejo de tecnologías, la capacitación, la readaptación de procesos empresariales y de recursos humanos como clave de innovación y competencia de las industrias, incluso a un nivel internacional, claro que en conjunto con un nivel de inversión y conocimiento en el área. 37

Es decir, los retos que se presentan ante la implementación de la industria 4.0 se presentan a continuación en cinco puntos principales:

- Productividad: debido a los años de funcionamiento y al seguimiento de estándares o procedimientos arraigados las empresas han presentado una baja productividad.

- Adaptación de los sistemas: se interpreta mediante la encuesta aplicada a las empresas lácteas que la adopción de sistemas inteligentes y tecnológicos ha sido un problema desde el costo hasta el proceso de implementación.

- Falta de capacitación del personal en el área tecnológica.

- Niveles de transformación y costos de inversión elevados de tecnologías.

- Deficiencia en equipos tecnológicos.

A su vez, los retos a los que se enfrentaron las empresas evaluadas, se ven reflejadas en factores internos, los cuales han sido subestimados $\mathrm{y}$, por lo tanto, han reflejado un reto mayor para las empresas en el momento de implementar la industria 4.0 a sus procesos. Estos retos internos se presentan enlistados de acuerdo al grado de anomia presentado en las empresas de estudio:

- Falta de conocimientos y habilidades en el área de tecnología.

- Capital humano: Se identificó que el capital humano es de edad avanzada en mayor porcentaje.
- Nula infraestructura especializada y adaptada a la industria 4.0.

- Falta de capacitación y costos de inversión incosteables.

- Deficiencia en departamentos de especialización.

- Deficiencia de maquinaria y herramientas tecnológicas para el proceso de distribución.

- Adopción de modelos de negocio actualizados y escalables adecuados a la empresa.

Contrariamente, los beneficios que han presentado las empresas encuestadas después de la implementación parcial o total de los avances tecnológicos, se presentan en los siguientes cuatro puntos; maquinaria, los costos, la productividad de la empresa, incremento del capital y las ganancias.

- Reducción de costos: se debe a que la inversión, a pesar de ser un riesgo por la cantidad y solvencia económica de la empresa, al ser amortizable con el ahorro de costos, la optimización correcta y la flexibilidad de los procesos productivos y de transporte reducen ventajosamente el costo final por producto.

- Maquinaria: requiere de una inversión inicial, dependerá del tamaño de la empresa, así como de su catálogo de productos, la cantidad de inversión y mantenimiento requerido, sin embargo, el implementar tecnología brindará interconexiones, disminución de tiempos, reducción de errores, y serán un soporte futuro para aumentar su producción y la calidad del producto final.

- Productividad y solución de errores: es necesario implementar tecnologías que permitan a la empresa aumentar su productividad y a su vez solucionar los errores operativos de manera automatizada y sin incidencias negativas.

- Aumento considerable y notorio de la economía y ventas que genere la empresa en comparación a la capacidad de la competencia.

- Transporte: la capacidad de producto que se puede transportar a largas distancias permitirá a la empresa tener una oportunidad ventajosa de expansión de mercados e incluso la movilidad del mismo en diversos Estados.

Con la finalidad de dar sustento a los retos y oportunidades que presentan las empresas lácteas en el proceso de implementar los avances tecnológicos de la industria 4.0, se elaboró una tabla para representar el nivel de inversión en ciencia, tecnología e innovación en México, esto con datos publicados en el Ranking Nacional de Ciencia, Tecnología e Innovación publicado en un periodo de evaluación del año 2008 al 2011, (Ver 
Tabla 2) (Foro Consultivo Científico y Tecnológico, 2011). 38

\begin{tabular}{|l|c|c|c|c|}
\hline $\begin{array}{c}\text { Entidad } \\
\text { Federativa }\end{array}$ & $\begin{array}{c}\text { Ciencia, } \\
\text { Tecnología e } \\
\text { Innovación }\end{array}$ & $\begin{array}{c}\text { Productividad } \\
\text { Innovadora }\end{array}$ & $\begin{array}{c}\text { Infraestructura } \\
\text { Empresarial }\end{array}$ & $\begin{array}{c}\text { Tecnologías de la } \\
\text { Información y } \\
\text { Comunicación }\end{array}$ \\
\hline $\begin{array}{l}\text { Distrito } \\
\text { Federal }\end{array}$ & 0.8961 & 1 & 0.391 & 1 \\
\hline Nuevo León & 0.3266 & 0.5079 & 0.8358 & 0.5779 \\
\hline Morelos & 0.2439 & 0.3452 & -0.1059 & 0.2119 \\
\hline Jalisco & 0.2433 & 0.1064 & 0.1201 & 0.4185 \\
\hline Coahuila & 0.1428 & 0.0093 & 0.2578 & 0.2185 \\
\hline Querétaro & 0.1188 & 0.4441 & 1 & 0.1986 \\
\hline $\begin{array}{l}\text { Baja } \\
\text { California }\end{array}$ & 0.0607 & -0.5514 & 0.1577 & 0.6523 \\
\hline Guanajuato & 0.0465 & 0.023 & 0.6212 & -0.1246 \\
\hline Chihuahua & 0.0298 & -0.021 & 0.0883 & 0.2331 \\
\hline $\begin{array}{l}\text { Estado de } \\
\text { México }\end{array}$ & 0.0178 & -0.2589 & 0.2686 & 0.1963 \\
\hline $\begin{array}{l}\text { Baja } \\
\text { California Sur }\end{array}$ & 0.0091 & -0.0948 & 0.3667 & 0.487 \\
\hline $\begin{array}{l}\text { Aguascaliente } \\
\text { S }\end{array}$ & -0.0374 & -0.5312 & 0.2534 & 0.2576 \\
\hline $\begin{array}{l}\text { San Luis } \\
\text { Potosí }\end{array}$ & -0.0667 & -0.396 & 0.0486 & -0.255 \\
\hline Puebla & -0.0795 & 0.0636 & -0.277 & -0.3403 \\
\hline Colima & -0.1019 & -0.4392 & 0.1676 & 0.2403 \\
\hline Tamaulipas & -0.1071 & -0.1794 & -0.1166 & 0.1077 \\
\hline Sinaloa & -0.1183 & -0.6503 & -0.2937 & 0.1536 \\
\hline Michoacán & -0.1276 & -0.576 & -0.0775 & -0.3276 \\
\hline Yucatán & -0.162 & 0.0108 & -0.2985 & -0.1817 \\
\hline Sonora & -0.1947 & -0.1276 & -0.0292 & 0.3767 \\
\hline Veracruz & -0.2088 & -0.6721 & -0.5336 & -0.4091 \\
\hline Nayarit & -0.2182 & -1 & -0.0886 & -0.0253 \\
\hline Quintana Roo & -0.3082 & -0.472 & -0.808 & 0.0175 \\
\hline Campeche & -0.3254 & -0.621 & -0.5774 & -0.2365 \\
\hline Hidalgo & -0.3556 & -0.6897 & 0.1284 & -0.5183 \\
\hline Tlaxcala & -0.3642 & -0.5023 & -0.3029 & -0.4005 \\
\hline Durango & -0.386 & -0.5533 & -0.3253 & -0.0666 \\
\hline Zacatecas & -0.4485 & -0.8417 & -0.0738 & -0.2384 \\
\hline Chiapasco & -0.5486 & -0.8435 & -1 & -0.5088 \\
\hline-0.5838 & -0.9518 & -0.8623 & -1 \\
\hline Guarrero & -0.8101 & -0.9759 & -0.9716 & -0.6347 \\
\hline
\end{tabular}

Tabla 2. Ranking de posiciones de los Estados ante el

Ranking Nacional de Ciencia, Tecnología e Innovación (2008-2011)

Fuente: Elaboración propia con datos del Ranking Nacional de Ciencia, Tecnología e Innovación 2008 -2011 (2020).
Al realizar la tabla comparativa se pudo observar que el Estado de Hidalgo no cuenta con un nivel favorable dentro de los rubros de inversión en Ciencia, Tecnología e Innovación, Productividad Innovadora y TIC'S, lo que refleja la cantidad de retos y rezago de avances tecnológicos en sus procesos de recolección, producción y sobre todo distribución.

Así mismo, podemos visualizar que a menor inversión pública, apoyos gubernamentales y capital empresarial la Infraestructura empresarial se ve afectada y por lo tanto disminuida, garantizando las desventajas comerciales de dichas empresas.

Contrariamente, se visualiza que el Estado de Hidalgo cuenta con un potencial competitivo en materia de Infraestructura empresarial, esto quiere decir que las empresas cuentan con un el equipo, la infraestructura física y el personal necesario para competir en un mercado, sin embargo las encuestas realizadas a las empresas evaluadas en la región de Tulancingo, Hidalgo, demuestran que el contar con maquinaria, equipo, infraestructura y personal no garantizan que sean una empresa competitiva, debido a que no cuentan con las habilidades para satisfacer un mercado nacional, su rezago tecnológico ha demostrado a nivel Estado, que se cuenta simplemente con los requisitos, aunque básicos, necesarios para competir y laborar en un mercado como el lácteo.

De igual manera, los resultados de la encuesta reflejaron que no se cuenta con Infraestructura TI la cual es, a grandes rasgos, el equipamiento adecuado que posee la empresa de cara al mercado productivo al que pertenece y así poder servir al cliente, por lo tanto, la posibilidad de que estas empresas cuenten con infraestructura en Tecnologías de la Información y Comunicación (TIC) es deficiente.

Es en este punto donde podemos relacionar los retos y las oportunidades con los factores externos propiciados por el gobierno que pueden entorpecer la implementación de la industria 4.0, los cuales, en un escenario estatal son un limitante de gran importancia para las empresas lácteas evaluadas.

\section{CONCLUSIONES}

De acuerdo con José Luis de la Cruz, director del Instituto para el Desarrollo Industrial y el Crecimiento Económico (IDIC), "México no está preparado para que la mayoría de las industrias implementen los avances mínimos requeridos para transitar hacia una industrialización 4.0 dentro de sus cadenas de suministro", (De la Cruz, 2019), la razón es que durante 25 años el país no cuenta con una política industrial, y con ello, un desarrollo industrial fuerte, lo que refleja un retraso notorio en innovación tecnológica de crecimiento. 39 
De igual manera, podemos observar en el indicador del INEGI que mide la productividad de la industria (IMAI), en el año 2019, registró un total de 10 meses de caídas consecutivas en el índice de productividad nacional, (INEGI, 2019). 40

Es posible pero incierto pensar que las micro y pequeñas empresas puedan desenvolverse en un futuro cercano dentro de las tecnologías y avances que integran esta Industria, sin embargo, y a pesar de los constantes factores que retrasan esta implementación, en las tabulaciones presentadas dentro de este trabajo de investigación podemos notar que existe una posibilidad de crecimiento y avance tecnológico, con la debida inversión y apoyo gubernamental.

Se visualiza que la falta de infraestructura, el uso de tecnologías obsoletas, la poca solvencia económica, así como el nulo conocimiento de los beneficios de los avances tecnológicos son sólo algunos elementos que han limitado el desarrollo económico y tecnológico de las empresas lácteas dentro de esta región. Al recabar la información proporcionada por las cinco empresas encuestadas se establece que:

Al menos el $60 \%$ de las empresas lácteas en la región no cuentan con un mercado de gran amplitud que exija automatizaciones o implementaciones tecnológicas para el aumento de producto distribuido, así mismo, se localizan en puntos cercanos a la empresa productora, lo cual refleja para los dueños una idea de "perdida de dinero" la inversión en equipo de rastreo y mejora de sus medios de transporte.

De igual manera, mediante el análisis de la encuesta aplicada, se considera que el costo de automatización de su equipo es elevado para su capacidad económica; la capacitación y el conocimiento que se tiene en materia tecnológica no es competente; se llegó a la conclusión que los factores externos que entorpecen la implementación de la industria, principalmente los apoyos gubernamentales para maquinaria de alta tecnología, son las barreras a las que las empresas en la región de Tulancingo se enfrentan constantemente por lo que han subestimado la importancia que esta implementación representa.

Por otro lado, las empresas que han implementado tecnología en su maquinaria, dispositivos de transporte y rastreo, entre otros, aseguran que sus puntos de venta se han triplicado y expandido a puntos diversos a la región de fábrica. Es en este punto que, mediante el análisis de las capacidades que son requeridas para competir en materia de tecnología, innovación, comunicación, productividad e infraestructura (TI, TIC, 4.0,entre otras), se puede determinar que las empresas lácteas evaluadas no tienen los avances tecnológicos necesarios para competir en mercados nacionales, por lo que se considera necesario la capacitación del sector lácteo y la introducción de métodos escalables y personalizados para cada una de las empresas lácteas, es fundamental resaltar que de acuerdo a los resultados obtenidos, no todas las empresas lecheras requieren de implementaciones tecnológicas en sus procesos, debido al volumen de producción y el sector al que se dirigen, es decir, sus objetivos.

De igual manera, se determina que las empresas con rezago tecnológico tienen un alto índice de desventaja en su producción, por lo que integrar avances tecnológicos en su departamento de distribución se considera innecesario en este momento, es por esta razón que se sugiere desarrollar la cadena de suministro en una cadena de valor e implementar las tecnologías pertinentes a dicho desarrollo.

Al considerar los resultados obtenidos de las encuestas se determina que no están dadas las condiciones para que las PYME's incursionen en su totalidad en la industria 4.0, sobre todo por la falta de financiamiento. Es necesario cubrir en primera instancia con las necesidades salariales de tal manera que la economía mejore y esto no sea un freno para la automatización de las industrias mexicanas. Posteriormente, se requiere un avance tecnológico nacional, implementación de los avances industriales y eliminar el rezago nacional en estas áreas.

\section{REFERENCIAS}

[1] Deloitte Touche Tohmatsu Limited. Forces of change: Industry 4.0. Rev. Deloitte Touche. 2017: 1-45.

[2] Del Valle, M. La industria lechera en los estados del norte, desarrollo y limitaciones. Estudios Fronterizos. 1986; 4(10): 11-42.

[3] Cuevas-Reyes, V. Diagnóstico de la cadena productiva de leche de vaca en el estado de Hidalgo. Técnica Pecuaria en México. 2007; 45(1): 25-40.

[4] Romero-Santillán, F. y otros. Demandas tecnológicas y de política para mejorar la competividad de la cadena agroaliemtaria deleche en el estado de Hidalgo. Revista Mexicana de Agronegocios. , 2009; 13(24): 774-787.

[5] Cervantes-Escotoa, F., Cesín Vargasb, A. \& Mamani Oño. La calidad estándar de la leche en el estado de Hidalgo. Revista Mexicana de Ciencias Pecuarias. 2013. 4(1): 75-86.

[6] Loera, J. \& Banda, J. Industria lechera en méxico: parámetros de la producción de leche y abasto del mercado interno. Revista de Investigaciones Altoandinas. 2017. 19(4): 419-426.

[7] Cardona-Arbeláez, D., Balza Franco, V. \& Henríquez Fuentes, G. Innovación en los procesos logísticos: Retos locales frente al desarrollo global. En: Universidad Libre, editors. 23 ed. Cartagena; 2017: 2-132.

[8] Huartos-Carranza, E. Importancia del aprovechamiento de la logística 4.0 y sus aportes al proceso de distribución de última milla. En: Universidad Militar Nueva Granada; 2019: 2-18.*

[9] Oliván-Cortés, R. La Cuarta Revolución Industrial, un relato desde el materialismo. Rev. de Estudios Urbanos y Ciencias Sociales. 2014; 6(2): $101-111$. 
[10] Espejel-García, A, Barrera Rodríguez, A. \& Cuevas-Reyes, V. Dinámica de la innovación y ganancias. Revista Electrónica Nova Scientia. 2015. 8(17): 391- 408.

[11] Medina-Rivera, R. \& Villegas-Valladares, E. FINANCIAMIENTO DE LA CIENCIA, LA TECNOLOGÍA Y LA INNOVACIÓN EN LAS REGIONES DE MÉXICO. Revista Mexicana de Agronegocios. 2016. 38(1): 253-270.

[12] SCHWAB, K. La Cuarta Revolución Industrial. En: DEBATE,editors. 1 ed. Barcelona; 2016: 100-149.

[13] Ynzunza Cortés, C. y otros. El Entorno de la Industria 4.0: Implicaciones y Perspectivas Futuras. Rev. CONCIENCIA TECNOLÓGICA. 2017; 54: 33-45.

[14] Cohen, Y. y otros. Assembly system configuration through Industry 4.0 principles:. ScienceDirect. 2017; 50(1): 14958- 14963.

[15] Taliaferro, A., Guenette, C., Awargal, A. \& Pochon, M. Industry 4.0 and distribution centers. Rev. Deloitte University Press. 2018: 1-20.

[16] Silva Casas, R. \& Estrada Dominguez, J. Capacidades dinámicas en las mipymes. Latindex. 2019: 60-70.

[17] Bearzotti, L. Industria 4.0 y la Gestión de la Cadena. Gaceta Sansana. 2017; 3(8): 12-48.

[18] Baco, A. I., Beliz, G., Coatz, D. \& Garnero, P. Industria 4.0 Fabricando el Futuro. Buenos Aires: Paidós. 2018.

[19] Arteaga, F. La cuarta revolución industrial (4RI): un enfoque de seguridad nacional. ELCANO. 2018: 2-32.

[20] Calatayud, A. \& Katz, R. Cadena de suministro 4.0: Mejores prácticas internacionales y hoja de ruta para América Latina. Estados Unidos: Banco Interamericano de Desarrollo. 2019.

[21] Cámara de Comercio de España. Foro "La transformación digital: la oportunidad para, Madrid. 2017

[22] WeFORUM (Schwab, K.) . The Global Competitiveness Report $2017-$ 2018. Columbia University. 2017.

[23] IMCO. ÍNDICE DE COMPETITIVIDAD ESTATAL 2016, México. 2016.

[24] KPMG; CEOE. Perspectivas del sector industria, España. 2018.

[25] Álvarez, A. Por qué la Industria 4.0 es un imperativo competitivo para la empresa. $2018 \quad$ [En Available at: https://cic40.es/por-que-la-industria-4-0-es-un-imperativocompetitivo-para-la-empresa/

[Último acceso: 22 Noviembre 2019].

[26]Cuevas Reyes, V. Diagnóstico de la cadena productiva de leche de vaca en el estado de Hidalgo. Técnica Pecuaria en México. 2007; 45(1): 2540.

[27] Romero Santillán, F. y otros. Demandas tecnológicas y de política para mejorar la competividad de la cadena agroaliemtaria deleche en el estado de Hidalgo. Revista Mexicana de Agronegocios. 2009; 13(24): 774-787.

[28] Cuevas Reyes, V. Diagnóstico de la cadena productiva de leche de vaca en el estado de Hidalgo. Técnica Pecuaria en México. 2007; 45(1): 2540.
[29] Amador Hernández, J. Estímulos fiscales para promover la inversión en ciencia, tecnología e innovación. Pluralidad y Consenso del Instituto Belisario Domínguez. 2017; 7(33): 415-430.

[30] Oficina de Información Científica y Tecnológica para el Congreso de la Unión,. Inversión para Ciencia, Tecnología e Innovación en México, Ciudad de México. 2018..

[31] [32] WeFORUM (Schwab, K.). The Global Competitiveness Report 2017-2018, Columbia University. 2017.

[33] Foro Consultivo Científico y Tecnológico. Ranking Nacional de Ciencia, Tecnología e Innovacion, México. 2018.

[34] Loera, J. \& Banda, J. Industria lechera en méxico: parámetros de la producción de leche y abasto del mercado interno. Revista de Investigaciones Altoandinas. 2017; 19(4): 419-426.

[35] Miebach, J. \& Ribas, J. Automatización en la manipulación de materiales. Énfasis Logística.. 2017:121-130.

[36] Taliaferro, A., Guenette, C., Awargal, A. \& Pochon, M.. Industry 4.0 and distribution centers. Deloitte University Press. 2018: 1-20.

[37] Deloitte Touche Tohmatsu Limited. Forces of change: Industry 4.0, Av. Paseo de la Reforma 505, Cuauhtémoc, 06500 Ciudad de México, CDMX: Deloitte Touche. 2017.

[38] Foro Consultivo Científico y Tecnológico. Ranking Nacional de Ciencia, México. 2011.

[39] De la Cruz, J. México y su retraso en la Cuarta Revolución Industrial. 2019. [Entrevista] (Octubre 2019).

[40] INEGI. Índice Nacional de Competitividad, México. 2019. 
ANEXO 1. Cuestionario de implementación "Industria 4.0"

Nombre de compañía-empresa

Instrucciones: Indique con una $X \mathrm{la}(\mathrm{s})$ casilla (s) con las que aplica (n) su empresa.

- En caso de que no aparezca la opción que aplique a su empresa, indicar la que aplique en la opción "OTROS".

\begin{tabular}{|c|c|c|c|c|}
\hline \multicolumn{2}{|c|}{ Integración de sensores: } & \multicolumn{2}{|c|}{ Intercambio de datos: } & Distribución de lotes pequeños: \\
\hline$\square$ & No se utilizan & $\square$ & Sin funciones & $\square$ Rígidos y pocas piezas \\
\hline$\square$ & Integrados & $\square$ & Funcion de identificacion & $\square$ Flexibles y adecuables \\
\hline$\square$ & Desconozco & $\square$ & $\begin{array}{l}\text { Intercambio de datos } \\
\text { (QR, entre otros) }\end{array}$ & $\square$ Distribución personalizada \\
\hline$\square$ & Otro: & $\square$ & Otro: & $\square$ Otro: \\
\hline \multicolumn{2}{|c|}{ Interfaz persona-máquina: } & \multicolumn{2}{|c|}{ Infraestructura TIC: } & exión áreas-departamentos: \\
\hline$\square$ & $\begin{array}{l}\text { Sin dispositivos } \\
\text { de comunicación }\end{array}$ & $\square$ & Datos inalámbricos & $\begin{array}{l}\square \text { Conexión mediante } \\
\text { aplicaciones digitales }\end{array}$ \\
\hline$\square$ & $\begin{array}{l}\text { Dispositivos de } \\
\text { visualización local }\end{array}$ & $\square$ & $\begin{array}{l}\text { Uso de internet en } \\
\text { maquinaria }\end{array}$ & $\begin{array}{l}\square \text { Conexión persona- } \\
\text { persona }\end{array}$ \\
\hline$\square$ & $\begin{array}{l}\text { Uso de } \\
\text { aplicaciones }\end{array}$ & $\square$ & $\begin{array}{l}\text { Portal con internet para } \\
\text { seguimiento de } \\
\text { pedidos }\end{array}$ & $\square$ Sin conexión/ áreas \\
\hline$\square$ & Otro: & $\square$ & Desconozco & $\square$ Otro: \\
\hline \multicolumn{2}{|c|}{ Conexión máquina-máquina: } & \multicolumn{2}{|c|}{ Procesamiento de datos: } & nunicación-conectividad: \\
\hline$\square$ & $\begin{array}{l}\text { Comunicación a } \\
\text { través de internet }\end{array}$ & $\square$ & $\begin{array}{l}\text { Almacén de } \\
\text { información }\end{array}$ & $\begin{array}{l}\square \text { Envío y recepción con uso } \\
\text { de internet }\end{array}$ \\
\hline$\square$ & Sin comunicación & $\square$ & $\begin{array}{l}\text { Sin procesamiento de } \\
\text { datos }\end{array}$ & $\begin{array}{l}\square \text { Envío y recepción sin uso } \\
\text { de internet }\end{array}$ \\
\hline$\square$ & Desconozco & $\square$ & $\begin{array}{l}\text { Control y almacén } \\
\text { mediante aplicaciones }\end{array}$ & $\begin{array}{l}\square \text { Sin comunicación- } \\
\text { conectividad }\end{array}$ \\
\hline$\square$ & Otro: & $\square$ & Otro: & $\square$ Otro: \\
\hline
\end{tabular}

Almacenamiento de datos: Monitoreo de unidad/producto: Servicios TI asociados a la distribución:

$\square \quad$ No se utilizan
$\square \quad$ Integrados
$\square \quad$ Desconozco
$\square \quad$ Otro:

$\square$ Sin monitoreo
$\square$ Monitoreo básico (Teléfono,
mensajes SMS, etc)
$\square$ Monitoreo mediante
aplicaciones
$\square$ Desconozco

Servicios a través de

portal online, apps, entre

otros.

$\square \quad$ Integrados a unidades

$\square$ Desconozco

Modelo de negocio actualizado a nuevas tecnológias

No conozco de las tecnologías y

prefiero no usarlas

Considero no necesitar este servicio

No cuento con capital para invertir

Considero pertinente su aplicación

y buscaré asesoramiento 